PAPER

\section{Application of encapsulated superabsorbent polymers in cementitious materials for stimulated autogenous healing}

To cite this article: Jani Pelto et al 2017 Smart Mater. Struct. 26105043

View the article online for updates and enhancements.
Related content

\begin{tabular}{l} 
Self-healing mortar with pH-sensitive \\
Superabsorbent polymers: testing of the \\
sealing efficiency by water flow tests \\
Elke Gruyaert, Brenda Debbaut, Didier \\
Snoeck et al. \\
- Sealing of cracks in cement using \\
\hline microencapsulated sodium silicate \\
P Giannaros, A Kanellopoulos and A Al- \\
Tabbaa \\
- Acrylate-endcapped polymer precursors: \\
effect of chemical composition on the \\
healing efficiency of active concrete cracks \\
Maria Araújo, Kim Van Tittelboom, Peter \\
Dubruel et al.
\end{tabular}




\title{
Application of encapsulated superabsorbent polymers in cementitious materials for stimulated autogenous healing
}

\author{
Jani Pelto ${ }^{1}$, Markku Leivo ${ }^{1}$, Elke Gruyaert ${ }^{2}$, Brenda Debbaut ${ }^{2}$, \\ Didier Snoeck ${ }^{2}$ and Nele De Belie ${ }^{2,3}$ \\ ${ }^{1}$ VTT Technological Research Centre of Finland Ltd, Vuorimiehentie 3, FI-02044 Espoo, Finland \\ ${ }^{2}$ Magnel Laboratory for Concrete Research, Ghent University, Tech Lane Science Park, Campus A, \\ Technologiepark Zwijnaarde 904, 9052 Ghent, Belgium \\ E-mail: nele.debelie@ugent.be
}

Received 16 March 2017, revised 14 July 2017

Accepted for publication 7 August 2017

Published 18 September 2017

\begin{abstract}
Superabsorbent polymers have shown potential for use in mortar and concrete as self-healing agents. The main drawback is, however, that these superabsorbent polymers also absorb mixing water during the preparation and casting of mortar or concrete, leading to a loss in workability. To avoid the absorption of mixing water, superabsorbent polymers were coated using a fluid bed spraying process. The barrier coating consisted of three successive coating layers:

polyvinylbutyral as primer/wetting layer, cyclo-olefin copolymer as a barrier layer and a sol-gel derived zirconium-silicon oxide as an adhesion-promoting topcoat layer. The coated SAPs were characterized, and their swelling determined to quantify the delay in uptake of water and $\mathrm{Ca}(\mathrm{OH})_{2}$ solution. The last was considered as the most important, as the SAPs will finally be applied in mortar or concrete having a pore solution with high $\mathrm{pH}$. The results showed that swelling could be delayed to a large extent, but for a short time. Results showed that the selfsealing efficiency of mortars was not affected by coating the SAPs. Moreover, due to the reduced uptake of mixing water, the strength reduction, noticed when uncoated SAPs were added to the mortar, could partly be compensated.
\end{abstract}

Keywords: superabsorbent polymers, coating, self-healing, mortar

(Some figures may appear in colour only in the online journal)

\section{Introduction}

The idea of using encapsulated and 'on-demand' activating self-healing additives in concrete has been described already some years ago, by for example Bho et al [1] and Yang et al [2], and reviewed by Van Tittelboom and De Belie [3], and Snoeck and De Belie [4]. However, commercial encapsulated self-healing agents have not emerged to the market, likely due to the lack of feasible processes for generating them, hampered by a potentially high price and/or a low volume of production.

\footnotetext{
3 Author to whom any correspondence should be addressed.
}

Crosslinked superabsorbent polymers (SAPs) are industrially available in large quantities. As such, they have been proposed as additives for concrete [5-9], and especially also for self-healing purposes [10-14]. Self-healing based on SAPs stems from their property of absorbing a large amount of liquids, thus filling the volume in cracks when a fluid intrudes into the cracks. The swelling capacity of dry SAP hydrogels is typically several hundred times their own weight, and the process is reversible: the hydrated SAP gel shrinks to its original dimension upon drying and is able to re-swell. This large swelling capacity can be potentially utilized by two useful mechanisms: in the sealing of cracks from water flow, and further augmenting the self-healing capacity by stimulation of cement hydration and in situ carbonation of the 
hydrated cement gel within the cracks in the concrete. The most feasible way of applying the SAP hydrogels in the selfhealing approach for cementitious materials is by dispersing the non-hydrated particles during concrete mixing. However, as SAPs readily take up part of the mixing water and swell during mixing and casting, they can generate macro-pores in the hardened concrete. To alleviate this drawback, the SAP particles are preferably encapsulated into a water-impermeable shell. The shell material should facilitate good mixing of the coated SAP particles in aqueous concrete mixes and mortars, and promote adhesion to the cement matrix. Moreover, the shell material should withstand the mechanical stresses of mixing, yet be fragile enough to break when a propagating crack passes the SAP particle.

Fluid bed spray coating is an attractive and up-scalable process for coating solid particles larger than roughly $50 \mu \mathrm{m}$ in size [15-18]. Particularly, there are clear benefits compared to wet chemical encapsulation methods, such as interfacial polymerization. The SAPs remain mostly dehydrated, and the particle size does not change significantly during slow spray coating in dry fluid beds, which is a benefit over working in wet phase, as the dense and brittle coating membrane would not withstand large dimensional changes without cracking during wetting-drying cycles. Furthermore, fluid bed spraying generates very little waste when a solvent recovery system is used. This is a clear benefit over wet chemical methods when considering up-scaling to multi-ton applications. To further justify the choice of fluid bed processing, similar technology has been successfully applied for decades in the batch and continuous coating of powders in the chemical, pharmaceutical, food and mineral processing industries.

Bottom spray fluid bed coating is undisputedly a very well controlled process for applying polymeric membranes onto solid powders. However, there are still several technical challenges in achieving liquid-tightness in spray coated polymer membranes on SAP particles. It has been generally recognized that, besides the coating formulation, the coating process conditions also play an important role in determining the final quality of the coating [19]. Hence, fluid bed coating processes generally require extensive process optimization. A central role in the performance of the coated SAPs is played by the water barrier property and the suitability of the coating material in the spray process. For the application in selfhealing concrete, excellent water barrier properties, stability at the high $\mathrm{pH}$ of cement slurry, and furthermore, solubility in water or suitable organic solvents is required.

In this study, a new water barrier polymer cyclo-olefin copolymer (COC) was investigated for the first time in a concrete application. COC is an amorphous hydrophobic thermoplastic polymer. It is well soluble, and hence well processable in tetrahydrofurane solvent (THF) at room temperature, and has water barrier properties outperforming the most common barrier (packaging) materials, such as semicrystalline polyolefins, e.g. high density polyethylene (HDPE), biaxially oriented polypropylene (PP) and polystyrene (PS). Generally, many industrial polymers, like the PS, offer chemical stability in high $\mathrm{pH}$, favourable mechanical properties and solubility to solvents (THF), but they have limited water vapour barrier properties. A simple calculation on water diffusion in polymers suggests that even for a high water barrier polymer such as COC with a water vapour permeability $<0.2 \mathrm{~g} \mathrm{~m}^{-2} /$ day, at least a $20 \mu \mathrm{m}$ thick layer of the barrier is necessary in order to achieve sufficiently long water retention times to assist the mixing of SAPs into concrete.

Coating of the hydrophilic SAP particle with a uniform, thick membrane of hydrophobic barrier polymer faces the inherent challenge of wetting and spreading of the coating. This can be alleviated by applying a more hydrophilic primer/wetting agent before applying the barrier polymer. However, even then, the great challenge remains in depositing a very thick ( $>20-30 \mu \mathrm{m})$, virtually defect-free membrane on the SAP. In fact, a single pinhole or a small crack in the protective coating will facilitate excess water uptake and fast swelling of the SAP particle during the early stages of the concrete mixing. A driving force to the water absorption is the osmotic pressure of the ionic SAP gel. This force is very powerful, especially in pure water, and in many cases results in the irreversible failure of the barrier coating within seconds. However, the extent of swelling is dependent on the $\mathrm{pH}$ and ionicity of the aqueous phase [20]. Typically, the swelling is significantly reduced in ionic media, which facilitates the processing of SAPs in cement slurry with moderate $(<100 \%)$ swelling during the mixing. However, the barrier coating is usually beneficial, as the major disadvantage of uncoated SAPs in fresh mortar/concrete mix is that they still absorb large quantities of mixing water, leading to unwanted effects-for example, loss of workability and macro-pore formation-especially in systems with a high water-to-cement ratio [21, 22].

The optimum size, shape, loading and distribution of the encapsulated self-healing material has recently gained increasing attention from researchers [3, 23-27]. Intuitively, small (micron-sized) particles, for example fine SAP particles, have better distribution in the matrix, and are hence more effectively encountered by propagating cracks. However, small particle size does not favour crack filling. Moreover, from the viewpoint of encapsulation, the smaller the particles are, the more encapsulating material will be needed to achieve protection from mixing water. Moreover, the behaviour of SAP particles in the fluidized bed coating process is highly dependent on the particle size. Fluidization properties of the particles according to the mass density and particle size follow the so-called Geldart group classification [28]. According to the classification, stable fluidization is normally achievable for polymeric non-cohesive particles larger than $50 \mu \mathrm{m}$ in diameter, and with mass densities around 1000-1400 $\mathrm{kg} \mathrm{m}^{-3}$ (Geldart Group A powder). Commercially available SAP powders prepared by bulk polymerization followed by crushing into $100 \mu \mathrm{m}$ to $500 \mu \mathrm{m}$ particle size ranges fall conveniently into this group, and can hence be coated using the fluid bed spray coating process. The current investigation concentrated on the crushed SAPs, which are non-optimally prismatic shaped containing sharp edges. However, the spray coating technique presented here can be extended to SAP 
particles in other size ranges (e.g. 50-100 $\mu \mathrm{m}$ ) and round shapes, as long as they can be fluidized.

\section{Encapsulation of superabsorbent polymers}

\subsection{Materials}

The ground SAP powder used in the current study was a commercially available SAP Floset $27 \mathrm{cc}$ supplied by SNF SAS (Andrezieux - France). Before fluid bed coating, the fine particle fraction (roughly $<100 \mu \mathrm{m}$ ) of the SAP powder was removed either by jet air classification (Hosakawa Alpine, Multi processing system) or by sieving (Frisch Analysette 3 PRO, mesh $80,<180 \mu \mathrm{m}$ ). The mean particle size (D50) was determined by laser diffraction in air, and amounted to $343 \mu \mathrm{m}$ and $381 \mu \mathrm{m}$, respectively, for the air classified sample and the sieved $(>180 \mu \mathrm{m})$ sample.

The organic solvents utilized in the fluid bed spraying process were $95 \%$ ethanol and tetrahydrofurane (THF). The polymers were sprayed from $10 \mathrm{w}-\%$ solutions.

The barrier coating consisted of three successive coating layers: a first layer of polyvinylbutyral (PVB, Kuraray Mowital B30H) as a primer/wetting layer, a second layer of cyclo-olefin copolymer (COC, Topas) as a barrier layer and a third layer of sol-gel derived zirconium-silicon oxide $\left(\mathrm{ZrSiO}_{2}\right)(\mathrm{VTT})$ as an adhesion-promoting topcoat layer.

\subsection{Fluid bed coating process}

2.2.1. Equipment. The fluid bed coating process was set up with an Aeromatic Fielder (nowadays GEA Pharma) Multiprocessor system (MP-1). The optimum batch size was $1.5 \mathrm{~kg}$ of SAP powder. The MP-1 system was equipped with a 12 litre stainless steel coating chamber, as shown in figure 1.

The operation in the coating process was adjusted by controlling the fluidizing air volume $\left(\mathrm{m}^{3} \mathrm{~s}^{-1}\right)$ and temperature, and the liquid feed rate of the polymer coating solution by a precision plunger metering pump (Prominent MikroDelta (B). These two variables control the rate of evaporation of the solvent. For a stable coating process, $+46{ }^{\circ} \mathrm{C}$ and $+55^{\circ} \mathrm{C}$ fluidizing air temperatures were utilized for the THF and ethanol solution, respectively. The feed rate of the spray solution was typically $120-200 \mathrm{ml} \mathrm{h}^{-1}$, corresponding to $12-20 \mathrm{~g} \mathrm{~h}^{-1}$ of the solid polymers. The air volume was adjusted continuously during the several hours of the coating process to prevent agglomeration of particles and blocking of the flow in the reactor.

2.2.2. Solvent borne coatings. Research on encapsulation of SAPs was a trial-and-error process, seeking optimal layer thicknesses for each of the polymers. During preliminary tests, different layer compositions were tested (tables 1 and 2). In the final batches, typically $8 \mathrm{w} \%$ of PVB polymer, $12 \mathrm{w} \%$ of $\mathrm{COC}$ coating and $3-4 \mathrm{w} \%$ of the dry $\mathrm{ZrSiO}_{2}$ xerogel was deposited on the SAP (values relative to the mass of the final coated product). Hence, this product contained in total $23-24 \mathrm{w}-\%$ of the coating materials
$\left(\mathrm{PVB}+\mathrm{COC}+\mathrm{ZrSiO}_{2}\right)$, corresponding to a theoretical coating thickness of $20-25 \mu \mathrm{m}$. The coating process was carried out in three successive steps: first, the primer polymer (PVB) in ethanol solution was sprayed on the SAP. After the desired amount of coating solution had been applied, pumping the solution was discontinued and the fluid bed kept running for a 15-30 min drying step. Subsequently, COC polymer in THF solvent was applied onto the SAP-PVB powder at $15-20 \mathrm{~g} \mathrm{~h}^{-1} \mathrm{COC}$ deposition rate. Hourly, short drying steps (5-10 min) were necessary during COC coating, to avoid agglomeration of the particles. After the application of the COC, the product was fully dried in the fluid bed over 30 min. Finally, the PVB and COC coated SAP was subjected to zirconium-silicon sol-gel spray solution in the fluid bed (VTT). After removal of the powders from the fluid bed, they were briefly heated to $+120^{\circ} \mathrm{C}$ in an oven.

\section{Characterization of the coated SAPs}

\subsection{Morphology of the coated SAP}

The appearance of the uncoated SAP particle, and the $\mathrm{PVB} / \mathrm{COC} / \mathrm{ZrSiO}_{2}$ coated SAP particle are depicted in the SEM images figures 2(A), (B)/(C), respectively. Figure 2(C) shows that the coating is uniform on the several SAP particles presented in the image. White arrows in the upper left corner of figure 2(C) indicate that there were some of the SAP particles fused together by the polymer coating. In the figure 2(B) a more detailed surface image of the coated SAP particle is shown. Comparing to the non-coated reference (2A), the surface coating was rough, containing homogeneously distributed craters and fine particles, appearing brighter in the image. The sharp edges of the prismatic SAP particles (figure 2(A)) had been rounded in shape, indicating that the coating covered the whole surface of the SAP particle. According to SEM/EDS elemental compositional point analyses, the outmost $\mathrm{ZrSiO}_{2}$ coating uniformly covered the polymer coating.

\subsection{Particle size of the coated SAP}

Particle size distribution (PSD) parameters of the coated SAP samples are summarized in table 1. PSDs were measured in air by the laser diffraction technique, using Malvern Mastersizer 3000. Mean particle diameters corresponding to the $10 \%, 50 \%$ and $90 \%$ percentiles are given. In order to facilitate the fluid bed processing, the fine particle fraction of the asreceived Floset $27 \mathrm{cc}$ powder was removed. Two alternative processes were investigated: at first, sieving was used (SAP_1, table 1), and in the later experiments, air classification (SAP_2, table 1) was used instead, as it is a significantly faster and preferred process compared to sieving in the $1.5 \mathrm{~kg}$ laboratory set up that was available. Coating experiments were done on both SAP_1 and SAP_2 powders. Comparing the PSDs of the two non-coated powders, it is evident that sieving is a more effective process for removing the fines. 

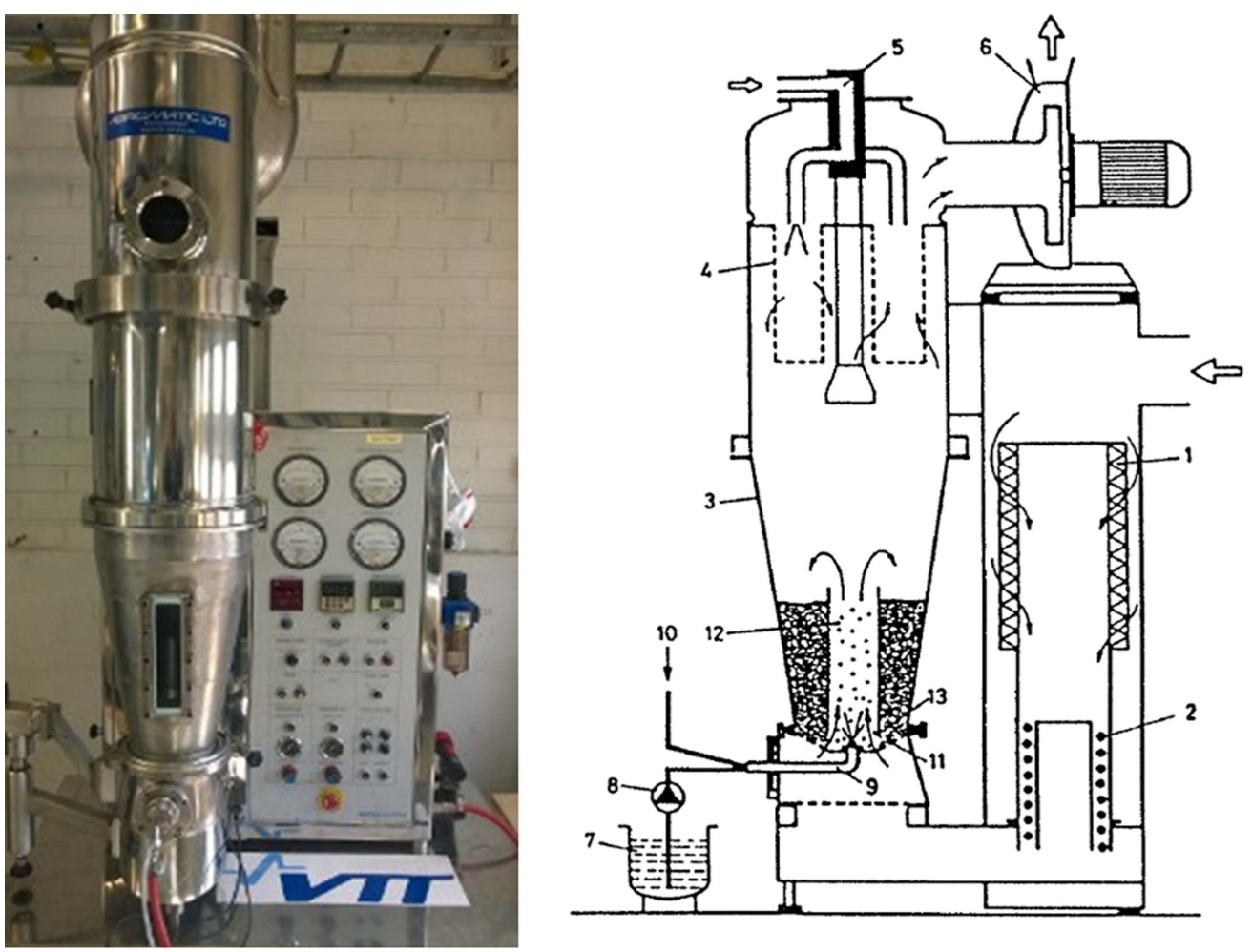

Figure 1. Left: Aeromatic Fielder Multiprocessor system (MP-1) utilized in the study. Right: The principle of the Wurster coating process is depicted in the schematic drawing; features include: 12 litre stainless steel coating chamber (3); stainless steel filter (4) to facilitate the processing of organic solutions (7) (original picture in the MP-1 operation manual).

Table 1. Particle size distribution (PSD) parameters of the polymer coated SAP samples. Mean particle diameters corresponding to the $10 \%, 50 \%$ and $90 \%$ percentiles. Data measured using Malvern Mastersizer 3000.

\begin{tabular}{lccc}
\hline Sample & $\begin{array}{c}\mathrm{D} 10 \\
(\mu \mathrm{m})\end{array}$ & $\begin{array}{c}\mathrm{D} 50 \\
(\mu \mathrm{m})\end{array}$ & $\begin{array}{c}\mathrm{D} 90 \\
(\mu \mathrm{m})\end{array}$ \\
\hline Floset 27cc & & & \\
SAP_1, sieved $(>180 \mu \mathrm{m})$ & 243 & 381 & 591 \\
SAP_2, air classified $(>100 \mu \mathrm{m})$ & 188 & 343 & 579 \\
21 w-\% PVB/COC on SAP_1 & 310 & 489 & 771 \\
24 w-\% PVB/COC $/ \mathrm{ZrSiO}_{2}$ & 339 & 467 & 644 \\
$\quad$ on SAP_2 & & & \\
\hline
\end{tabular}

Also, it was found that the coating process works equally for both the sieved and air classified Floset.

The particle sizes of the coated samples were significantly larger than the non-coated controls. The observed increase of the $50 \%$ percentile, corresponding to the peak maximum in the PSD, was $108 \mu \mathrm{m}$ and $124 \mu \mathrm{m}$ for the PVB/ $\mathrm{COC}$ and $\mathrm{PVB} / \mathrm{COC} / \mathrm{ZrSiO}_{2}$ coating on SAP_1 and SAP_2 respectively. These values are different from the expected coating thickness or the thicknesses derived by weighing/ calculation and SEM imaging, but rather reflect the quality of the coating process, in particular the agglomeration. For example, the true coating thickness of the $\mathrm{PVB} / \mathrm{COC} / \mathrm{ZrSiO}_{2}$ was roughly $20-25 \mu \mathrm{m}$. The $90 \%$ percentile was systematically increased in all coated samples, indicating agglomeration and fusing of particles. In fact, both the agglomeration and the spreading of the PSD, especially the smaller particle size tail, were both worse in the sample coated with the PVB/ COC $(21 \mathrm{w}-\%)$ on the SAP_1, despite the lower amount of deposited coating. This was most likely not due to the SAP preparation method but rather due to a better coating process control for the sample batch $\mathrm{PVB} / \mathrm{COC} / \mathrm{ZrSiO}_{2}(24 \mathrm{w}-\%)$ deposited on the SAP_2. The spreading on the PSD can be explained by the varying coating thickness of the different size particle fractions due to different flow behaviours in the fluid bed, and hence controlled by the process parameters. The sample $24 \mathrm{w}-\%$ PVB/COC $/ \mathrm{ZrSiO}_{2}$ on SAP_2 was prepared once more experience was acquired with the coating technique for SAPs.

The $10 \%$ percentile was also systematically increased in all the coated samples, indicating that also all the SAP particles had indeed been coated. In addition, no fine particle fraction below the D10 of the controls was generated in the PSD.

\subsection{Swelling of coated SAPS}

3.3.1. Fast swelling test. Absorption of water or saturated $\mathrm{Ca}(\mathrm{OH})_{2}$ aqueous solution by the SAP powders was assessed by a fast swelling test, using the filtration method. Briefly, $0.15 \mathrm{~g}$ of the SAP powder was mixed with $100 \mathrm{~g}$ of the aqueous solution in a beaker. The swelling was allowed to continue for four minutes. After that period, the SAP powder was filtrated off over approximately two minutes time in a conical funnel, using a filter paper. The mass uptake of the water or freshly prepared aqueous $\mathrm{Ca}(\mathrm{OH})_{2}$ solution per dry 

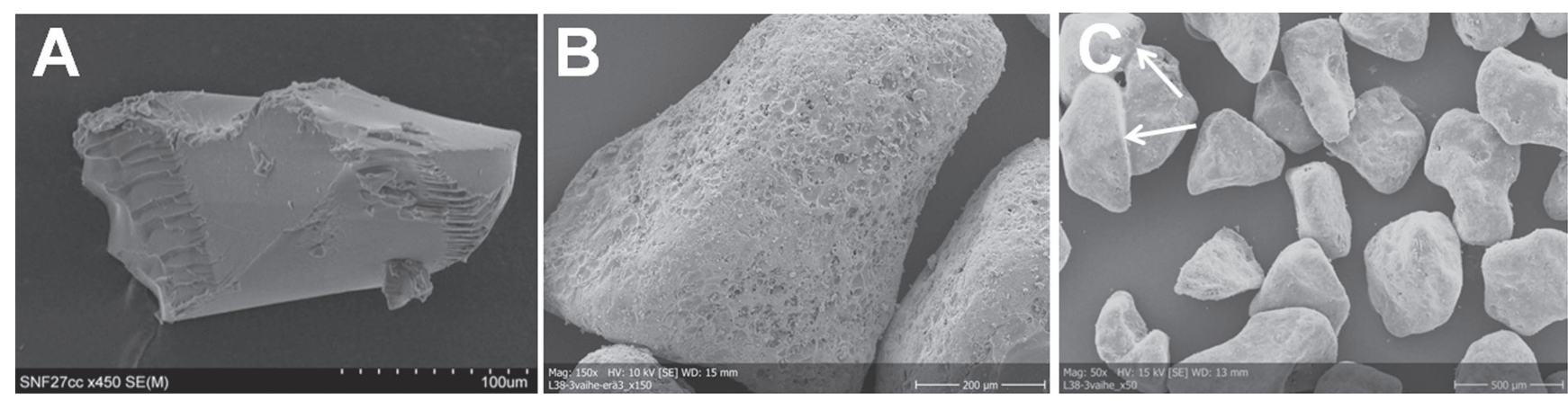

Figure 2. Representative SEM images of (A) Floset 27cc SAP hydrogel particle. (B) and (C); $\mathrm{PVB} / \mathrm{COC} / \mathrm{ZrSiO}_{2}$ coated Floset $27 \mathrm{cc}$.

Table 2. Swelling of coated SAP hydrogels in saturated $\mathrm{Ca}(\mathrm{OH})_{2}$ aqueous solution (six minutes test time). Some data points are also presented for swelling in de-ionized water. For the coated SAPs, the uptake of water is expressed per gram of coated SAP.

\begin{tabular}{lll}
\hline sample & SW in de-ionized water & SW in aqueous saturated $\mathrm{Ca}(\mathrm{OH})_{2}$ \\
\hline Floset 27cc SAP & $480 \mathrm{~g} / \mathrm{g}$ SAP & $86 \mathrm{~g} / \mathrm{g} \mathrm{SAP}$ \\
SAP + PVB $(6 \mathrm{w}-\%)+\mathrm{COC}(10 \mathrm{w}-\%)^{\mathrm{a}}$ & $250 \mathrm{~g} / \mathrm{g}$ coated SAP & $29-31 \mathrm{~g} / \mathrm{g}$ coated SAP \\
& $297 \mathrm{~g} / \mathrm{g}$ SAP & $35-37 \mathrm{~g} / \mathrm{g}$ SAP \\
\hline
\end{tabular}

Sequential (1-3) deposition of $\mathrm{PVB} / \mathrm{COC} / \mathrm{ZrSiO}_{2}$ layers (approximately $20 \mu \mathrm{m}$ total coating thickness $\left(\mathrm{PVB}+\mathrm{COC}+\mathrm{ZrSiO}_{2}\right)$

\begin{tabular}{lcl}
\hline (1) $\mathrm{SAP}+\mathrm{PVB}$ primer $(8 \mathrm{w}-\%)$ & n.a. & $35 \mathrm{~g} / \mathrm{g}$ coated SAP \\
& & $38 \mathrm{~g} / \mathrm{g}$ SAP \\
(2) $\mathrm{SAP}+\mathrm{PVB}$ primer $(8 \mathrm{w}-\%)$ & n.a. & $20 \mathrm{~g} / \mathrm{g}$ coated SAP \\
$+\mathrm{COC}(12 \mathrm{w}-\%)$ & & $25 \mathrm{~g} / \mathrm{g} \mathrm{SAP}$ \\
$\begin{array}{l}\text { (3) } \mathrm{SAP}+\mathrm{PVB} \text { primer }(8 \mathrm{w}-\%) \\
+\mathrm{COC}(12 \mathrm{w}-\%)\end{array}$ & n.a. & $10 \mathrm{~g} / \mathrm{g} \mathrm{SAP}$ \\
$+\mathrm{ZrSiO}_{2}(4 \mathrm{w}-\%)$ & &
\end{tabular}

preliminary test batch without the $\mathrm{ZrSiO}_{2}$ layer

weight of the SAP hydrogel is defined as the swelling (SW) of the hydrogel, and is expressed as grams of water/grams of SAP. The typical results are tabulated in table 2.

Swelling in pure water is always significantly faster and larger than in the ionic medium $\mathrm{Ca}(\mathrm{OH})_{2}$ (aq. sat.), as expected. Fast uptake of pure water, and hence the failure of the coatings, occurred in two minutes. The presence of the coating delays the water uptake in the test, as indicated by the difference in the measured swelling for the Floset $27 \mathrm{cc}$ (480 g/g SAP) and the PVB/COC coating (297 g/g SAP), but the numbers are both quite high. The coating will not significantly suppress the swelling capacity of the SAP particles in contact with pure water. Upon crack formation in mortar, the coating will thus not compromise the water sealing and self-healing capacity stimulated by the SAP. In concrete mixing, the SAP particles should preferably be added directly to high ionicity and high $\mathrm{pH}$ water (cement slurry), to avoid fast swelling. Tests executed in $\mathrm{Ca}(\mathrm{OH})_{2}$ medium show that the swelling is significantly slower for the coated SAPs. SAPs coated with approximately $6 \mathrm{w}-\%$ PVB primer and $10 \mathrm{w}-\%$ COC polymer had significantly lower uptake (36 g/g SAP) compared to the reference SAP $(86 \mathrm{~g} / \mathrm{g}$ SAP). Further improved results were obtained with higher amounts of deposited COC. Table 2 presents the results for samples taken during the sequential deposition of
$\mathrm{PVB} / \mathrm{COC} / \mathrm{ZrSiO}_{2}$ coating. It was noted that the $\mathrm{PVB}$ primer/wetting layer also had some barrier properties, but $12 \mathrm{w}-\%$ of $\mathrm{COC}$ and the sol-gel inorganic layer were necessary in order to reduce the swelling to the best achieved level of $10 \mathrm{~g} / \mathrm{g}$ SAP.

The swelling rate of uncoated and coated SAPs was also compared by monitoring the volume change of swollen SAPs in cement filtrate [29]. The cement filtrate was prepared by mixing $100 \mathrm{~g}$ of OPC (CEM I $42.5 \mathrm{~N}$ ) with $1 \mathrm{~L}$ of demineralized water and subsequent filtering. Results, as shown in figure 3 , indicate that the coating delays the uptake of the liquid. Nevertheless, swelling (in $\mathrm{g} / \mathrm{g}$ SAP) of the coated SAPs starts already after a few minutes and after $\sim 20 \mathrm{~min}$ the water uptake is comparable to that of uncoated SAPs.

3.3.2. Swelling under optical microscope. The sequence of stereomicroscope images in figure 4 shows the kinetics of swelling of the Floset 27cc SAP and the PVB/COC $/ \mathrm{ZrSiO}_{2}$ coated SAPs in saturated $\mathrm{Ca}(\mathrm{OH})_{2}$ solution. The three upper images show that the uncoated Floset SAP absorbs the liquid immediately after the pipetting. At $25 \mathrm{~s}$, all the SAP particles have already swollen to several times their original size. The bottom sequence of six images shows the swelling of the $\mathrm{PVB} / \mathrm{COC} / \mathrm{ZrSiO}_{2}$ coated SAP. At 25 and $55 \mathrm{~s}$ time points 


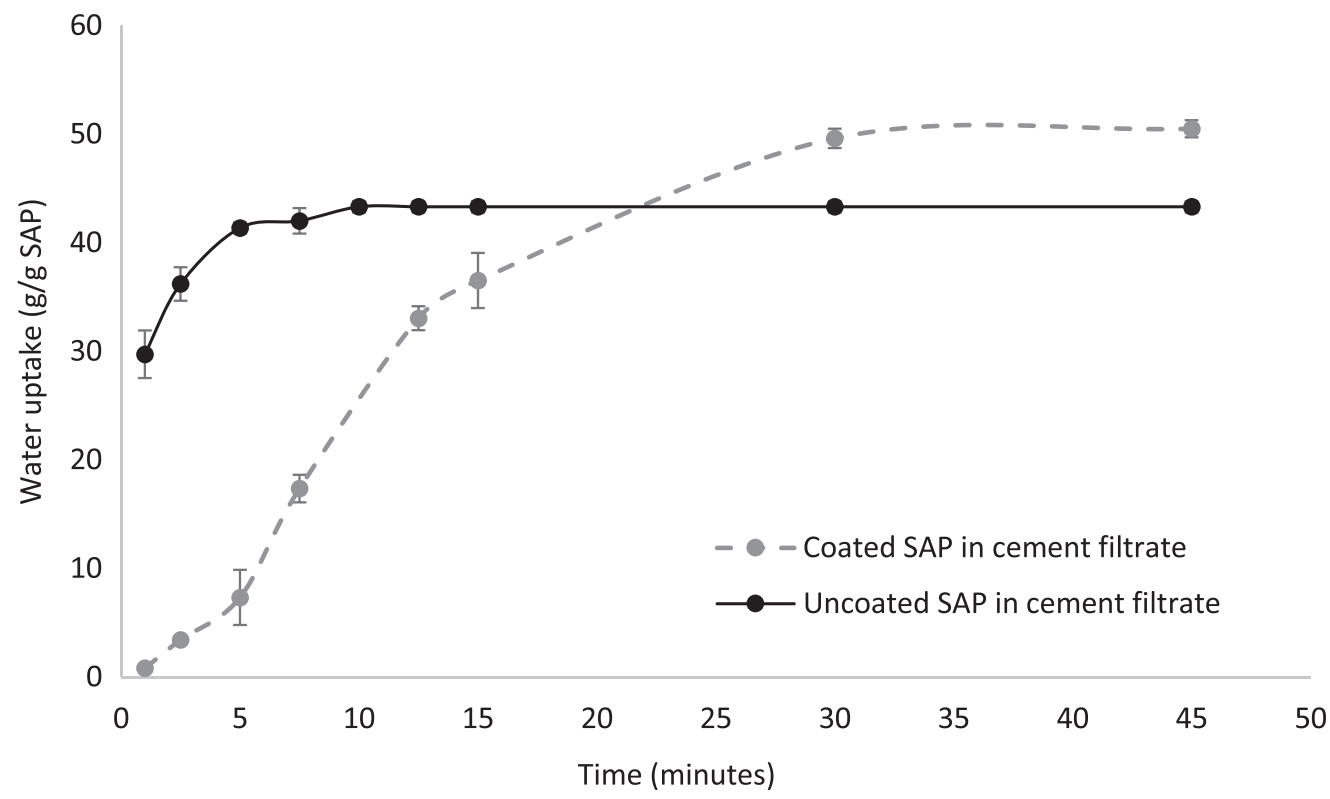

Figure 3. Swelling of coated and uncoated SAPs in cement filtrate solution as function of time. Swelling is expressed in g/g SAP for both the coated and uncoated SAPs.
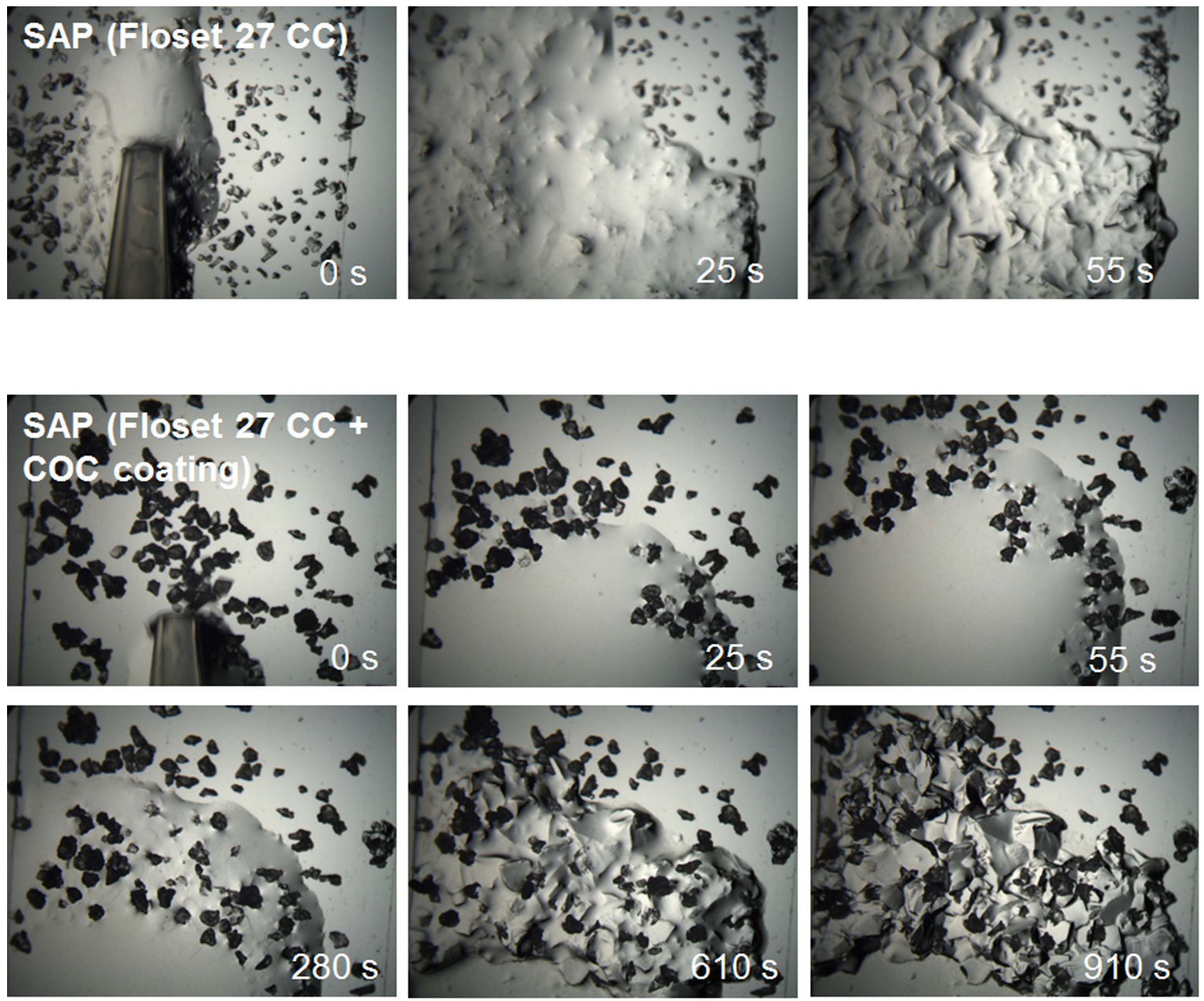

Figure 4. Sequence of stereomicroscope images depicting the swelling of Floset $27 \mathrm{cc} \mathrm{SAP}$ and $\mathrm{PVB} / \mathrm{COC} / \mathrm{ZrSiO}_{2}(8 / 12 / 4 \mathrm{w} \%) \mathrm{coated}$ SAP in saturated $\mathrm{Ca}(\mathrm{OH})_{2}$ solution. $200 \mu \mathrm{l}$ volume of the test solution was pipetted onto the powders at time point $0 \mathrm{~s}$. 


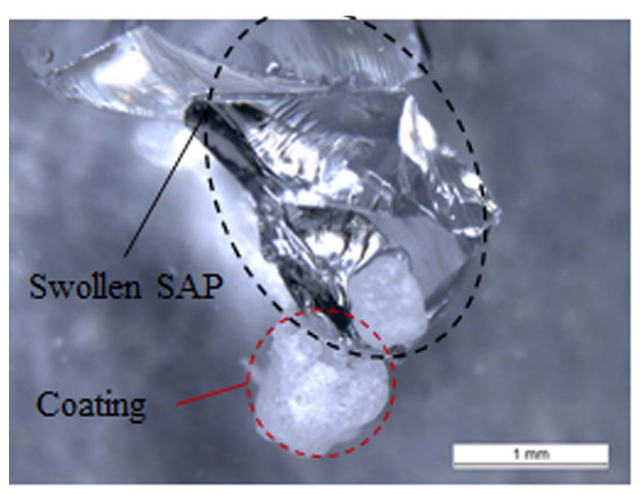

Figure 5. Swelling action of a coated SAP after rupture of the coating.

all the coated SAP particles are virtually unaffected. At $280 \mathrm{~s}$ some the coated particles show signs of swelling. It appears the shells, showing darker contrast in the images, have retained their shape, but the hydrogel has started to extrude out of the protective shell. Hence, the initial failure of the coating had occurred after a few minutes. At $610 \mathrm{~s}$ ( $\sim 10 \mathrm{~min})$, the process was completed, and the hydrogel had reached its full swollen volume.

When observing the swelling capacity by means of optical microscopy, the swelling of the coated SAP could be observed. When the coating was partly open at a location, the polymer was still able to swell to its full extent. This can clearly been seen in figure 5 where a swollen particle is swelling outside its coating (bottom whitish part). A dry particle was put under a microscope and a droplet of water was added. Due to a small crack feature in the coating, the SAP started to swell to full extent out of the coating. As shown in figure 5, it is as if the coating opened like a box, and the SAP came out.

\section{Application of coated SAP in mortar}

In literature, typical dosages of SAPs in mortar with a view to seal a crack from intruding fluids and to stimulate autogenous healing are mentioned, and vary in general from $0.5 \mathrm{~m} \%$ up to $1 \mathrm{~m} \%$ relative to the cement weight $[4,10-14,21,30]$. These amounts are quite high in comparison to the dosages needed for internal curing $(0.3 \mathrm{~m} \%$ to $0.6 \mathrm{~m} \%$ relative to cement weight) [5-9, 31], and it is thus expected that the effect on fresh and hardened properties cannot be neglected, especially for mixes with a high $w / c$ ratio $[22,32]$ as studied in this paper. For these mixes, the water absorbed by the superabsorbent polymers during mixing is not necessary to mitigate autogenous shrinkage due to the higher water-to-cement ratio, and as a consequence, macro-pore formation leading to a reduced strength of the matrix will be dominant over the positive effect of internal curing on the mechanical strength. The higher the amount of superabsorbent polymers is, the higher is the detrimental effect on the mechanical strength in these systems. The application of a coating should minimize these negative effects on the mechanical strength, while
Table 3. Mix composition of the reference mortar (REF), mortar with SAPs (SAP) and mortar with coated SAPs (C-SAP) (g).

\begin{tabular}{llll}
\hline & REF & SAP & C-SAP \\
\hline CEM I 42.5 N & 450 & 450 & 450 \\
Water & 225 & $225+90$ & 225 \\
Sand 0/2 & 1350 & 1350 & 1350 \\
SAP & & 4.5 & $4.5+1.35$ (coating) \\
\hline
\end{tabular}

maintaining the self-sealing and self-healing properties, as explained above. As the coating should prevent the SAPs from swelling upon contact with mixing water, ideally no additional mixing water should be added to compensate for the loss in workability. In that case, the effective water-tocement ratio (i.e. the water not absorbed by the SAPs, over the cement content) is the same and the same meso- and microstructural properties should be found for the cementitious matrix in between the coated SAP particles and the matrix of the mixture without SAPs [22, 33]. In literature, only one reference to coated SAPs in cementitious materials can be found. It is a gypsum-chitosan-gypsum coated SAP in well cement, for plugging micro cracks. The coating counteracted the loss in pumpability, strength and integrity of the cement [34].

In the current study, the suitability of the $\mathrm{PVB} / \mathrm{COC} / \mathrm{ZrSiO}_{2}$ coated SAPs for application in selfhealing mortar was evaluated. A comparison was made with a reference mortar (according to EN 196-1) and a self-healing mortar containing the same type of SAPs (Floset 27cc, SNF Floerger) without coating. The dosage of SAP (coating not included) added to the mix corresponded to $1 \mathrm{~m} \%$ by cement weight, i.e. the typical amount for self-sealing and self-healing purposes in cementitious materials. The mixing procedure applied was according to the Standard NBN EN 196-1 and the SAPs were added dry together with the cement, to ensure a homogeneous dispersion of the SAPs in the cement. Additional water was added to the mix with uncoated SAPs to compensate for the loss in workability, as was performed in earlier research [21]. Perfect behavior of the coating was assumed and no additional water was added to the mix with coated SAP. The mortar compositions can be found in table 3 .

\subsection{Effect on the fresh and hardened properties}

As SAPs are water absorbers, the effect of their addition on the consistency of mortar has been investigated by using the flow table. The procedure described in the Standard NBN EN 1015-3 was followed, and the flow values were determined immediately after mixing. This method seemed to be good to exactly determine the amount of mixing water absorbed, as was experimentally verified by means of image analysis of the macro-pores on polished cross-sections of specimens with the respective SAP used in this study [10, 21, 22]. The results tabulated in table 4 show that, for the mixes with untreated SAPs the additional water $(20 \mathrm{~g}$ water/g SAP) compensated for the loss in workability, while the coating cannot completely prevent the uptake of mixing water, leading to a 
Table 4. Flow value of mixes with and without SAPs.

\begin{tabular}{lc}
\hline Mix & Flow value $(\mathrm{mm})$ \\
\hline REF & 190 \\
SAP & 190 \\
C-SAP & 145 \\
\hline
\end{tabular}

decrease of the flow value. Nevertheless, all mixtures can be considered as 'plastic mortars' according to the classification of NBN EN 1015-6.

The effect of the addition of (coated) SAPs on the mechanical properties of mortar was determined by performing bending and compressive strength tests according to the Standard EN 196-1 on mortar prisms $40 \times 40 \times 160 \mathrm{~mm}^{3}$ at the age of 28 days. The storage condition as described in the Standard was used. The results are presented in table 5 . It is clear that simple addition of $1 \mathrm{~m} \%$ SAPs and additional water ( $20 \mathrm{~g}$ water/g SAP) leads to an unacceptable strength reduction $(>50 \%$ reduction of the compressive strength). By coating the SAPs, this strength reduction can be partly compensated $(<25 \%$ reduction of the compressive strength), but is still quite high. Furthermore, as the coated SAPs still absorb part of the mixing water, it is expected that the effective $\mathrm{w} / \mathrm{c}$ ratio is lower compared to the reference sample. It is, however, anticipated that (i) by selection of the most appropriate SAPs for coating (e.g. rounded shape), (ii) by improvement of the coating technique and (iii) by optimizing the mix design, the whole process can be optimized and the strength reduction can be minimized. However, when looking at the obtained results, the coating of the SAPs seems promising, to allow the inclusion of higher amounts of SAPs for self-sealing and self-healing compared to plain addition of SAPs, as the mechanical strength is reduced to a lower extent. However, the decrease is still pronounced, and further research will focus on how to coat the SAPs more effectively.

\subsection{Microscopic investigation of crack closure}

Prismatic mortar specimens $\left(40 \times 40 \times 160 \mathrm{~mm}^{3}\right)$ were cast, containing two reinforcement wires (diameter $1 \mathrm{~mm}$ ) and a hole over the length of the specimen (diameter $5 \mathrm{~mm}$ ) (see section 4.3). After curing for 28 days at $20{ }^{\circ} \mathrm{C}$ and $>95 \% \mathrm{RH}$, the specimens were cracked in a crack width controlled 3 -point bending test, so that the final crack mouth opening was in the interval $[50,200 \mu \mathrm{m}[$. Microscopic images were taken to determine the crack opening immediately after crack creation. As the crack width varies along the crack length, five measurements were taken and the mean value determined. Furthermore, additional measurements were taken after a period of 28 wet-dry cycles ( $12 \mathrm{~h}$ wet- $12 \mathrm{~h}$ dry) during which crack closure by $\mathrm{CaCO}_{3}$ precipitation and formation of hydration products was stimulated. Some representative pictures are given in figure 6 for crack widths around $100 \mu \mathrm{m}$ and $150 \mu \mathrm{m}$, showing that for the smaller crack widths, crack closure at the surface of the specimen is complete for all types of specimens, even the reference specimens, while for the
Table 5. Bending and compressive strength of mixes with and without SAPs (mean value \pm standard deviation).

\begin{tabular}{lcc}
\hline Mix & $\begin{array}{c}\text { Bending strength } \\
\left(\mathrm{N} \mathrm{mm}^{-2}\right)\end{array}$ & $\begin{array}{c}\text { Compressive strength } \\
\left(\mathrm{N} \mathrm{mm}^{-2}\right)\end{array}$ \\
\hline REF & $8.2 \pm 0.3$ & $53.0 \pm 1.7$ \\
SAP & $5.2 \pm 0.2$ & $24.6 \pm 0.7$ \\
C-SAP & $6.3 \pm 0.6$ & $40.4 \pm 1.8$ \\
\hline
\end{tabular}

larger crack widths, the presence of SAPs stimulates the autogenous healing capacity leading to an almost perfect crack closure within 28 wet-dry cycles for SAP and C-SAP specimens.

The products formed in the crack are most likely a combination of C-S-H, $\mathrm{CH}$ and $\mathrm{CaCO}_{3}$, as also found in literature $[11,12,14]$. These products are mainly formed due to autogenous healing and its promotion by superabsorbent polymers [35-39].

\subsection{Assessment of the self-sealing ability}

To assess the self-sealing ability, water flow tests as developed within the FP7 project HEALCON and described in Gruyaert et al [21] were performed. The principle is as follows: water, pressurized at 0.05 bar, is allowed to flow through the hole in the cracked prismatic mortar specimen. As the other end of the hole is sealed and the hole intersects with the crack, water will drip from the crack in case the crack is not (completely) closed, while no water outflow will be measured when the crack is sealed. Based on the water flow measurements $\left(W\right.$, in $\left.\mathrm{g} \mathrm{min}{ }^{-1}\right)$ immediately after crack formation and measurements on the same specimens after a healing period of 28 wet-dry cycles, the sealing efficiency $\left(S E_{\mathrm{WD}}\right)$ can be calculated:

$$
S E_{\mathrm{WD}}=\frac{W_{\mathrm{time}=0}-W_{\mathrm{time}}=28 d}{W_{\text {time }}=0} \times 100 \% .
$$

The results are presented in figure 7 and show the $S E_{\mathrm{WD}}$ as a function of the crack mouth opening. It is clear that up to a crack width of $\sim 125 \mu \mathrm{m}$, the autogenous healing capacity in reference specimens is sufficient to prevent water outflow from the hole via the crack to the surroundings. Only for larger crack widths is there a beneficial effect of the incorporation of SAPs. Except for one test result, it seems that cracks up to at least $175 \mu \mathrm{m}$ in mortars containing (coated) SAPs can be sealed for $>80 \%$ within the considered healing period. The coating of the SAPs does not seem to inhibit the proper functioning of the SAPs and the self-sealing ability of C-SAP mortars is comparable to that of SAP mortars. For crack widths higher than $150 \mu \mathrm{m}$, the sealing ability is however reduced, both for reference mortars and the mortars with (coated) SAP.

In addition to the water flow tests, a water leakage test was performed. The tests were executed with REF and C-SAP mortar specimens $\left(40 \times 40 \times 160 \mathrm{~mm}^{3}\right)$. The mortar mixes were the same as described in table 3 , except that the consistence of the C-SAP mixes was adjusted by adding extra 

$\sim 100 \mu \mathrm{m}$
$\sim 150 \mu \mathrm{m}$

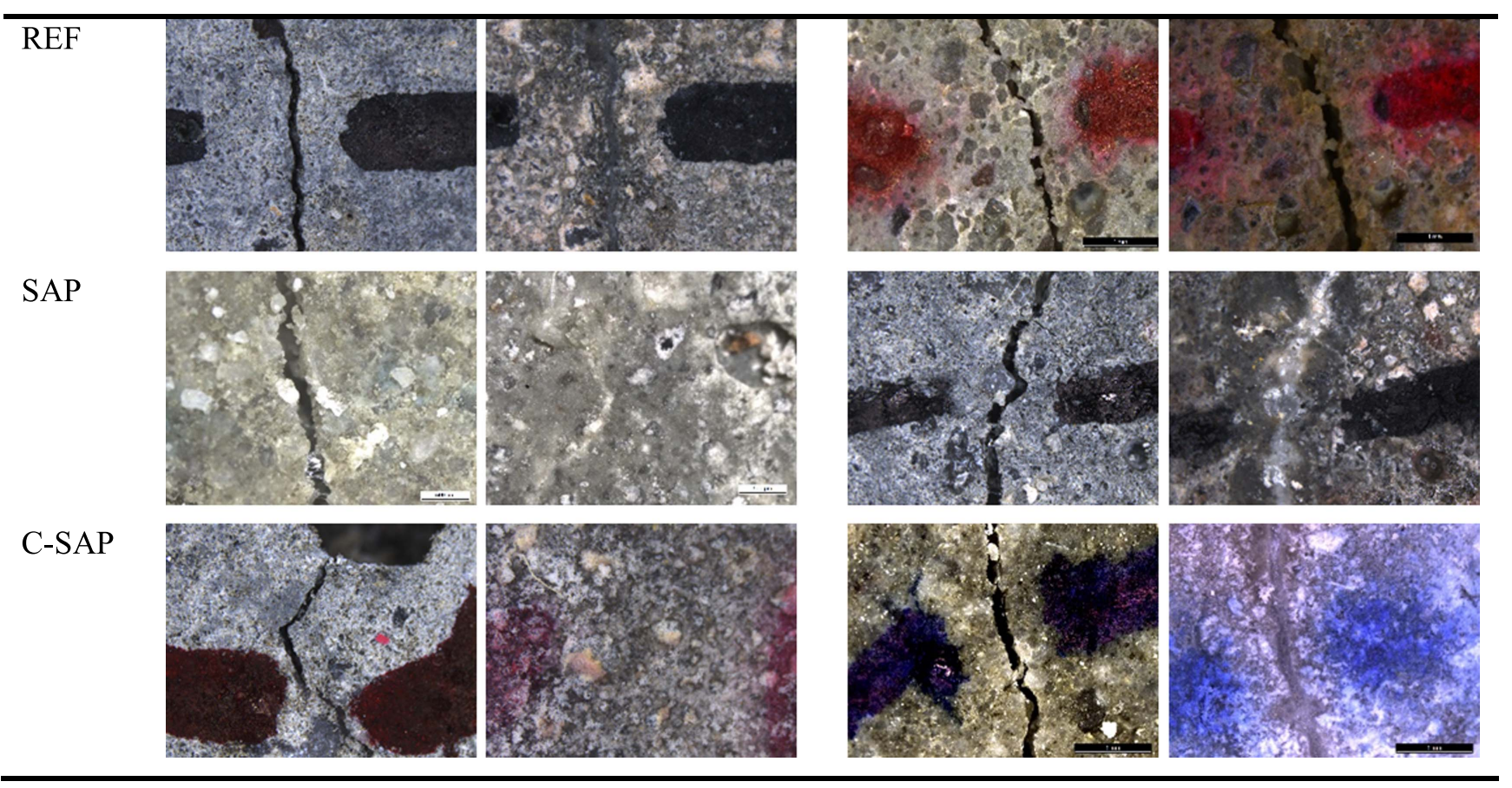

Before healing

After healing

Before healing

After healing

Figure 6. Optical microscopy images of cracks in mortar immediately after crack creation (left) and after exposure to 28 wet-dry cycles (right).

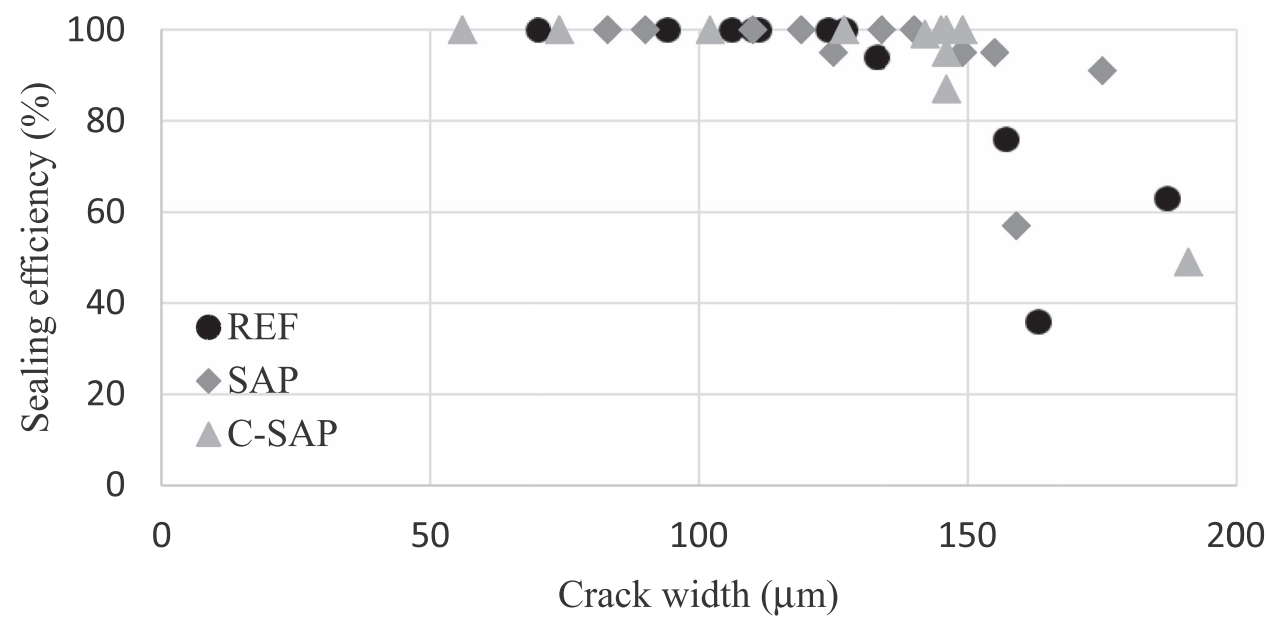

Figure 7. Sealing efficiency $\left(S E_{\mathrm{WD}}\right.$ ) as function of the original crack mouth opening (mean value) for REF, SAP and C-SAP specimens. The healing regime considered is 28 days with wet $(12 \mathrm{~h})$-dry $(12 \mathrm{~h})$ cycles.

water $(8 \mathrm{~g}$ water $/ \mathrm{g} \mathrm{SAP})$. In that way, the fresh mortar obtained almost the same workability as the reference mix (flow: $\sim 170 \mathrm{~mm}$ after 10-15 min). After 56 days moist curing, the samples were sawn into two pieces $\left(40 \times 40 \times 80 \mathrm{~mm}^{3}\right)$. These samples were split in two by bending. Small copper wires (size $0.05 \mathrm{~mm}, 0.15 \mathrm{~mm}$ or $0.4 \mathrm{~mm}$ ) were placed at both sides of the crack surfaces and the pieces were assembled by gluing aluminum sheets on their sides to support the formed crack. The real crack width was measured at ten spots along the crack at the top and bottom side of each specimen. A neoprene sheet was then glued on the outer surrounding of the specimen, to form a watertight reservoir on the top of the samples.

A water layer of $10 \mathrm{~mm}$ was introduced on top of the samples. Leakage through the crack was measured with an automatic balance. The tests were repeated during the same day (2-5 h later) and then three or four times until 28 days. In between the tests, the samples were moist-cured at $\sim 100 \%$ RH.

In figure 8 , the amount of water leaked out of the crack during a timeframe of $30 \mathrm{~min}$ is presented against the measured average crack width of the sample. For the $0.4 \mathrm{~mm}$ 


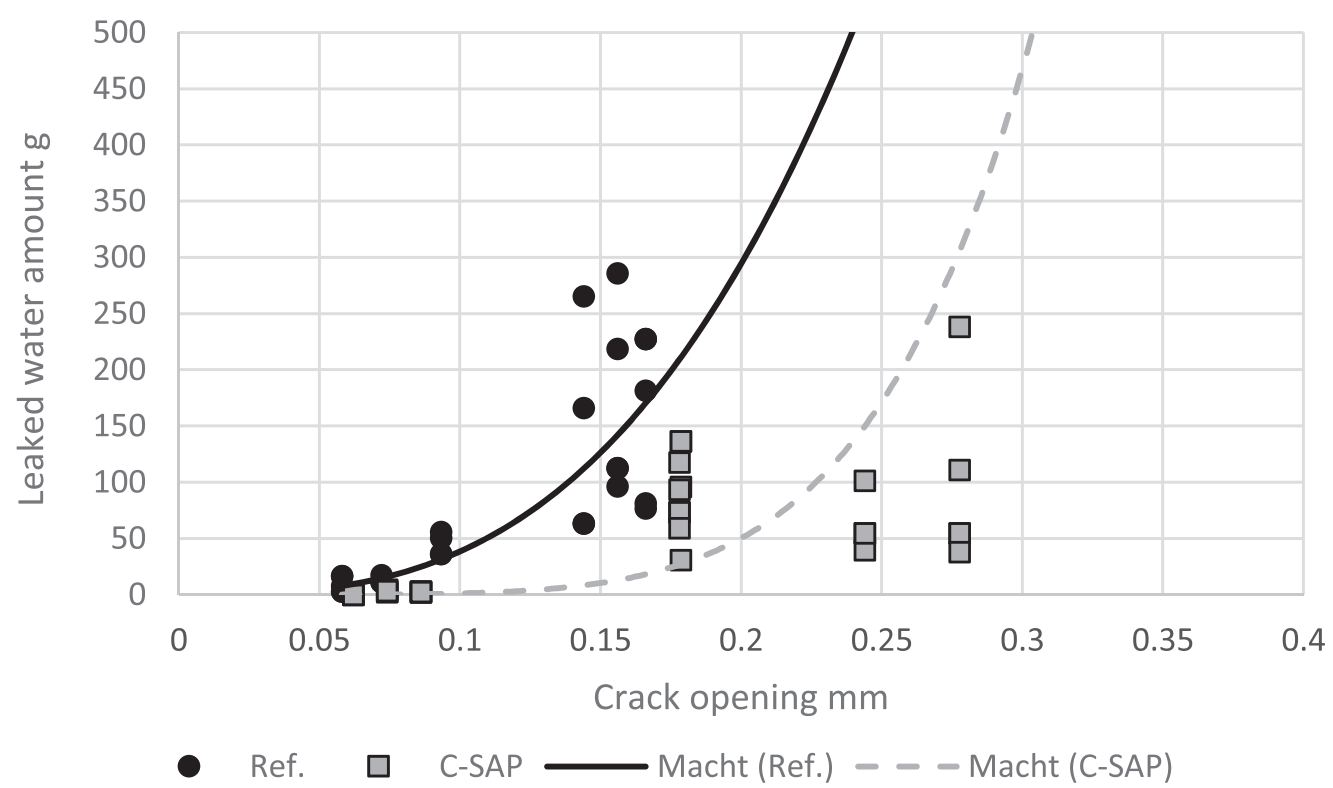

Figure 8. Amount of water leaked through the crack after $30 \mathrm{~min}$, considering cracks with different widths (blue dots: REF; red dots: C-SAP).
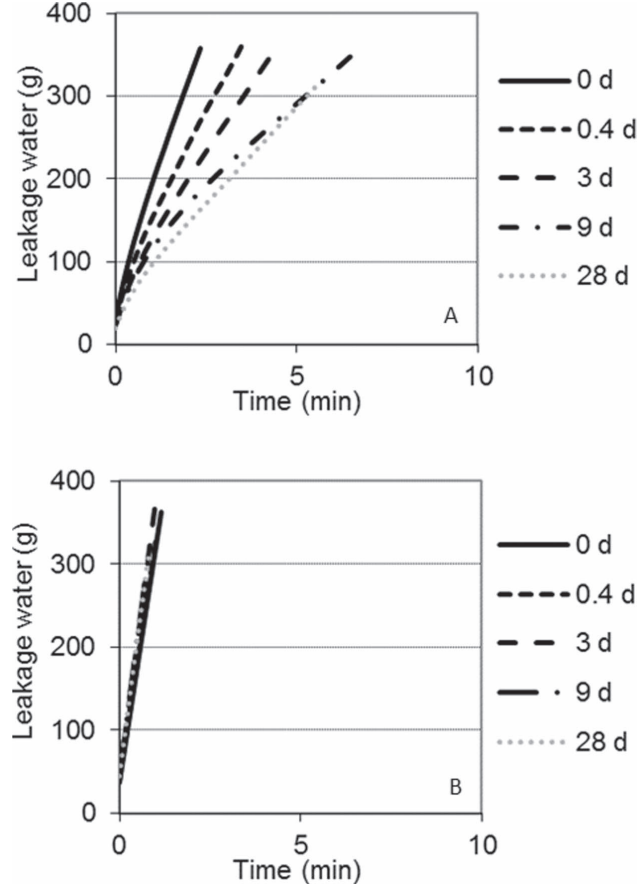

Figure 9. Evolution of the self-sealing of a large crack $(0.4 \mathrm{~mm})$ in function of time for mortar containing coated SAP (A) and for a reference specimen (B).

cracks in both the REF and the C-SAP specimens, the measurements were not accurate as the water leakage was very high and emptying the measuring system during the measurements disturbed the tests. As a consequence, these results are not presented.

The tests were executed the first day after assembling the specimens. From figure 8, it can be seen that coated SAP did not totally prevent the water leakage, but they have a very high effect to lower the leakage. Third power function regression lines have been added to visualize this effect as the water permeability is proportional to the third power of the crack width $[14,40]$. In the microscopic analysis, it was found that the SAP had penetrated only locally to the crack, not covering the whole crack surface.

After testing this immediate self-sealing effect due to swelling of the SAPs, also the evolution of the self-sealing in function of time due to (stimulated) autogenous healing was followed up by repeating the tests at later ages. In some of the samples (e.g. figure 9), the effect of stimulated autogenous healing on the self-sealing was clearly seen: the water leaked through the large crack of $0.4 \mathrm{~mm}$ in C-SAP mortar was considerably reduced as the curing time prolonged (figure 9(A)). No additional sealing was noticed in the reference sample due to autogenous healing (figure 9(B)). Moreover, with smaller crack sizes, a reduced water leakage was not always noticed so clearly. This may be due to the fact that even small openings in partially healed cracks may lead to a permeable composite, leading to the leakage of water. Moreover, as autogenous healing is dependent on the composition of the material (e.g. no healing near aggregates), the healing is some kind of patch healing.

With the test arrangement as presented here, quite some scatter on the results was obtained and further research will be necessary to draw clear conclusions.

\subsection{Scanning electron microscopy to study the macro porosity}

Different mortar specimens were broken into small pieces of approximately $1 \mathrm{~cm}$. The pieces were submerged in isopropanol for 7 days at an age of 28 days to remove the water in the system and to stop the cement hydration [41]. The solvent was renewed every day, with a solution:sample volume ratio of 10:1 [42]. The specimens were subsequently dried for another week in a vacuum container under low pressure $(\mathrm{P}<0.3 \mathrm{~Pa} ;-0.9$ relative to $1 \mathrm{~atm})$ and in the presence of silica gel. Finally, the specimens were dried in a ventilated oven at $40{ }^{\circ} \mathrm{C}$ until constant mass. The solvent 

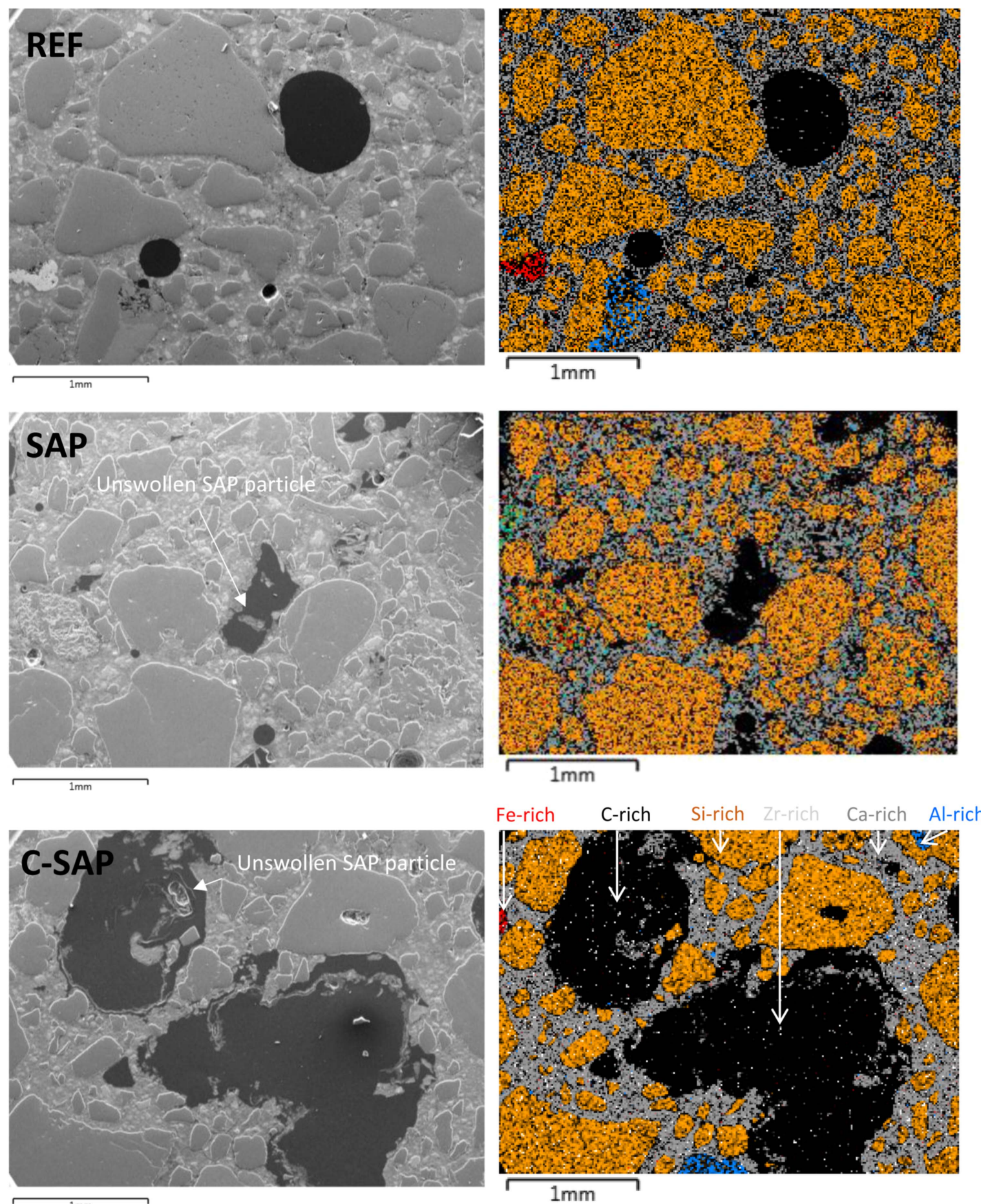

Figure 10. SEM images and EDX spectra of the studied specimens. EDX legend: yellow Si-rich, gray Ca-rich, blue Al-rich, red Fe-rich and white $\mathrm{Zr}$-rich regions next to black $\mathrm{C}$-rich regions. Please see the online version for the color diagrams.

exchange method followed by ambient drying is the best known method for preserving the fragile microstructure [41].

The specimens were impregnated using a low-viscosity epoxy resin. The epoxy was made by mixing $28 \mathrm{~g}$ of Hærder HY 2996 with $100 \mathrm{~g}$ of Conpox Harpiks BY 158. The impregnation was done under vacuum $(4 \mathrm{~h}$ at -0.9 relative to $1 \mathrm{~atm})$ to prevent entrapped air in the specimens' pieces and in the epoxy. The specimens were then stored at $40{ }^{\circ} \mathrm{C}$ for $48 \mathrm{~h}$ to allow curing and hardening of the epoxy. The specimens were subsequently ground on a rotating wheel using a $\mathrm{SiC}$ abrasive paper (No. 320-grit) and water until the particles became uncovered. Subsequently, the specimens were impregnated a second time, to ensure complete filling of all the pores with epoxy. The specimens were then ground for a final time with different grades of grit paper, to produce a completely smooth surface (accuracy of $0.25 \mu \mathrm{m}$ ) [43]: 
- SiC abrasive paper (No. 320-grit) and water at $300 \mathrm{rpm}$ until the same plane section became uncovered;

- SiC abrasive paper (No. 2400-grit) and DP-Lubricant Brown (Struers) at $300 \mathrm{rpm}$;

- Diamond paste (subsequently $3 \mu \mathrm{m}, 1 \mu \mathrm{m}$ and $0.25 \mu \mathrm{m}$ ) and isopropanol at $300 \mathrm{rpm}$;

- At the end, the specimens were cleaned with a MD Nap (soft cloth) to remove all impurities.

- The specimens were coated with a thin carbon layer of approximately $33.5 \mathrm{~nm}$, by means of a plasma magnetron sputter coater. The Scanning Electron Microscope (SEM) analysis was performed on a JEOL JSM-7600F instrument equipped with a detector for Energy-Dispersive $\mathrm{X}$-ray spectroscopy (EDX) analysis.

The results of the analysis are shown in figure 10. The microstructure of the mortar is clearly seen: Si-rich sand particles are surrounded by $\mathrm{Ca}$-rich paste regions originating from the hydration products. Only a few $\mathrm{Al}$ - and $\mathrm{Fe}$ - rich regions are found, and are caused by the type of aggregates. The surface was also covered by oxygen due to the presence of several oxides, but this was not depicted or computed in the figure itself as the distribution was homogeneous. The other elements were therefore studied. The black pores are also inherent to the studied system. For a cementitious matrix without SAPs (reference sample), the air voids appear to be round, as this is entrapped air. For the SAP specimens, irregular pores are also found. These are macro-pores formed due to the superabsorbent polymers. As the absorbed mixing water is only released during hardening, the SAPs leave behind macro-pores. If one closely examines the micrograph and the middle macro-pore, some remains of an un-swollen SAP particle can be found (left-hand side). It is composed out of C-rich regions, typical for a SAP particle and the epoxy-filled pores (right-hand side).

The specimen containing the coated SAPs also showed big macro-pores together with remaining SAPs and remaining coating. The Zirconium, of which the coating is composed, showed a uniform distribution and some localized dots near the interface of the macro-pore with the cementitious matrix. The $\mathrm{ZrSiO}_{2}$ layer was quite thin to measure with the EDX analysis. These points can be visible coating remnants, presumably from the Polyvinylbutyral primer or the cyclic olefin copolymer. The quantitative observation was, however, not possible, as the $\mathrm{Zr}$ concentration was too low compared to the accuracy of the measurement technique itself.

\section{Conclusion}

In this work, a method to encapsulate superabsorbent polymer (SAP) particles into a hydrophobic water barrier polymer cyclo-olefin copolymer (COC) shell, deposited from organic THF solvent, was demonstrated for the first time. The barrier coatings could prevent, to a large extent, the swelling of the SAP particles in saturated $\mathrm{Ca}(\mathrm{OH})_{2}$ for time periods relevant to the mixing of concrete. The coating survived the relevant mechanical loads during mixing in mortar using laboratory methods.

The best achieved barrier properties were obtained with a multi-layer PVB $/ \mathrm{COC} / \mathrm{ZrSiO}_{2}$ coating. The coating could be successfully applied on prismatic shaped SAP particles in a bottom spray fluid bed coating procedure involving three successive steps: application of a primer/wetting layer (PVB) from ethanol, COC barrier polymer from THF solvent and sol-gel derived $\mathrm{ZrSiO}_{2}$ adhesion-promoting layers. Approximately 20-25 $\mu \mathrm{m}$ average coating thickness, corresponding to approximately $24 \mathrm{w}-\%$ weight fraction based on the dry weight of the coated SAP, was needed to achieve significant reduction in the swelling in the fast swelling test (6 min). In cement filtrate solution, full absorption of the coated SAPs was delayed for approximately $20 \mathrm{~min}$.

As the coated SAPs were developed with a view to application in self-healing mortar and concrete, the effect on the fresh and hardened properties as well as on the selfsealing efficiency of cracks was determined. The results showed that, although the coating is not able to completely prevent swelling during the preparation of the mortar, the reduction of the strength properties by incorporation of SAPs could be partly compensated. Moreover, upon crack formation, the coated SAPs performed as well as uncoated SAPs with regard to crack sealing and crack healing, promoting autogenous healing of cracks. The work presented here serves as a basis for future research in this area.

\section{Acknowledgments}

The research leading to these results has received funding from the European Union Seventh Framework Program (FP7/2007-2013) under grant agreement $n^{\circ} 309451$ (HEALCON).

As a Postdoctoral Research Assistant of the Research Foundation-Flanders (FWO-Vlaanderen), Didier Snoeck wants to thank the foundation for its financial support.

\section{References}

[1] Bho B and Šumiga B 2008 Microencapsulation technology and its applications in building construction materials $R M Z$ Materials and Geoenvironment 55 329-44

[2] Yang Z, Hollar J, He X and Shi X 2009 Feasibility investigation of self-healing cementitious composite using oil core/silica gel shell passive smart microcapsules Proc. of SPIE-The Int. Society for Optical Engineering, Society of Photo-optical Instrumentation Engineers p 74934L

[3] Van Tittelboom K and De Belie N 2013 Self-healing in cementitious materials-a review Materials 6 2182-217

[4] Snoeck D and De Belie N 2015 From straw in bricks to modern use of microfibres in cementitious composites for improved autogenous healing-a review Constr. Build. Mater. 95 774-87

[5] Mechtcherine V and Reinhardt H-W 2012 Application of Super Absorbent Polymers (SAP) in Concrete Construction (Berlin: Springer) (https://doi.org/10.1007/978-94-0072733-5) 
[6] Mechtcherine V et al 2014 Effect of internal curing by using superabsorbent polymers (SAP) on autogenous shrinkage and other properties of a high-performance fine-grained concrete: results of a rilem round-robin test, TC 225-SAP Mater. Struct. 47 541-62

[7] Mechtcherine V et al 2017 Effect of superabsorbent polymers (SAP) on the freeze-thaw resistance of concrete: results of a RILEM interlaboratory test Mater. Struct. 50 1-19

[8] Jensen O M and Hansen P F 2001 Water-entrained cementbased materials I. Principles and theoretical background Cem. Concr. Res. 31 647-54

[9] Jensen O M and Hansen P F 2002 Water-entrained cementbased materials II. Experimental observations Cem. Concr. Res. 32 973-8

[10] Snoeck D 2015 Self-healing and microstructure of cementitious materials with microfibres and superabsorbent polymers Department Structural Engineering (Ghent: Ghent University) p 364

[11] Snoeck D and De Belie N 2015 Repeated autogenous healing in strain-hardening cementitious composites by using superabsorbent polymers J. Mater. Civ. Eng. 2804015086

[12] Snoeck D, Dewanckele J, Cnudde V and De Belie N 2016 Xray computed microtomography to study autogenous healing of cementitious materials promoted by superabsorbent polymers Cem. Concr. Comp. 65 83-93

[13] Snoeck D, Steuperaert S, Van Tittelboom K, Dubruel P and De Belie N 2012 Visualization of water penetration in cementitious materials with superabsorbent polymers by means of neutron radiography Cem. Concr. Res. 42 1113-21

[14] Snoeck D, Van Tittelboom K, Steuperaert S, Dubruel P and De Belie N 2014 Self-healing cementitious materials by the combination of microfibres and superabsorbent polymers J. Intel. Mat. Syst. Str. 25 13-24

[15] Teunou E and Poncelet D 2002 Batch and continuous fluid bed coating-Review and state of the art Journal of Food Engineering 53 325-40

[16] Jono K, Ichikawa H, Miyamoto M and Fukumori Y A 2000 Review of particulate design for pharmaceutical powders and their production by spouted bed coating Powder Technol. 113 269-77

[17] Christensen F N and Bertelsen P 1997 Qualitative description of the Wurster-based fluid-bed coating process Drug Development and Industrial Pharmacy 23 451-63

[18] Werner S R L, Jones J R, Paterson A H J, Archer R H and Pearce D L 2007 Air-suspension particle coating in the food industry: part I-state of the art Powder Technol. 171 25-33

[19] Laksmana F L 2010 Functional encapsulation of small particles; quantification and control of the morphology of the coating layers Faculty of Mathematics and Natural Sciences (Groningen: University of Groningen)

[20] Eichenbaum G M, Kiser P F, SImon S A and Needham D 1998 $\mathrm{pH}$ and ion-triggered volume response of anionic hydrogel microspheres Macromolecules 31 5084-93

[21] Gruyaert E, Debbaut B, Snoeck D, Díaz P, Arizo A, Tziviloglou E, Schlangen E and De Belie N 2016 Selfhealing mortar with $\mathrm{pH}$-sensitive superabsorbent polymers: testing of the sealing efficiancy of self-healig mortars by water flow tests Smart Mater. Struct. 25084007 084001-11

[22] Snoeck D, Schaubroeck D, Dubruel P and De Belie N 2014 Effect of high amounts of superabsorbent polymers and additional water on the workability, microstructure and strength of mortars with a water-to-cement ratio of 0.50 Constr. Build. Mater. 72 148-57

[23] Kanellopoulos A, Giannaros P and Al-Tabbaa A 2016 The effect of varying volume fraction of microcapsules on fresh, mechanical and self-healing properties of mortars Constr. Build. Mater. 122 577-93

[24] Kanellopoulos A, Giannaros P and Al-Tabbaa A 2016 Polymeric microcapsules with switchable mechanical properties for self-healing concrete-synthesis and proof of concept Smart Mater. Struct. 2645025

[25] Wang X, Sun P, Han N and Xing F 2017 Experimental study on mechanical properties and porosity of organic microcapsules based self-healing cementitious composite Materials 1020

[26] Dong B, Fang G, Ding W, Liu Y, Zhang J, Han N and Xing F 2016 Self-healing features in cementitious material with urea -formaldehyde/epoxy microcapsules Constr. Build. Mater. 106 608-17

[27] Mostavi E, Asadi S, Hassan M M and Alansari M 2015 Evaluation of self-healing mechanisms in concrete with double-walled sodium silicate microcapsules J. Mater. Civ. Eng. 2704015035

[28] Geldart D 1972 The effect of particle size and size distribution on the behaviour of gas-fluidised beds Powder Technol. 6 $201-15$

[29] Jensen O M 2011 Water absorption of superabsorbent polymers in a cementitious environment ed C K Y Leung and K T Wan Int. RILEM Conf. on Advances in Construction Materials through Science and Engineering (Hong Kong SAR: RILEM Publications S.A.R.L.) pp 22-35

[30] Van Tittelboom K et al 2016 Comparison of different approaches for self-healing concrete in a large-scale lab test Constr. Build. Mater. 108 125-37

[31] Snoeck D, Jensen O M and De Belie N 2015 The influence of superabsorbent polymers on the autogenous shrinkage properties of cement pastes with supplementary cementitious materials Cem. Concr. Res. 74 59-67

[32] Hasholt M T, Jensen O M, Kovler K and Zhutovsky S 2012 Can superabsorbent polymers mitigate autogenous shrinkage of internally cured concrete without compromising the strength ? Constr. Build. Mater. 31 226-30

[33] Snoeck D, Velasco L F, Mignon A, Van Vlierberghe S, Dubruel P, Lodewyckx P and De Belie N 2015 The effects of superabsorbent polymers on the microstructure of cementitious materials studied by means of sorption experiments Cem. Concr. Res. 77 26-35

[34] Liu H, Bu Y, Sanjayan J G, Nazari A and Shen Z 2016 The application of coated superabsorbent polymer in well cement for plugging the microcrack Constr. Build. Mater. 104 $72-84$

[35] Edvardsen C 1999 Water permeability and autogenous healing of cracks in concrete ACI Mater. J. 96 448-54

[36] Granger S, Loukili A, Pijaudier-Cabot G and Chanvillard G 2007 Experimental characterization of the self-healing of cracks in an ultra high performance cementitious material: mechanical tests and acoustic emission analysis Cem. Concr. Res. 37 519-27

[37] ter Heide N 2005 Crack healing in hydrating concrete Civil Engineering and Geosciences, Microlab (Delft: Delft University of Technology) p 128

[38] Homma D, Mihashi H and Nishiwaki T 2009 Self-healing capability of fibre reinforced cementitious composites J. Adv. Concr. Technol. 7 217-28

[39] Maes M, Snoeck D and De Belie N 2016 Chloride penetration in cracked mortar and the influence of autogenous crack healing Constr. Build. Mater. 115 114-24

[40] Tsukamoto M and Woener J D 1991 Permeability of cracked fibre-reinforced concrete Darmstadt Concrete 6 123-35

[41] Snoeck D, Velasco L F, Mignon A, Van Vlierberghe S, Dubruel P, Lodewyckx P and De Belie N 2014 The influence of different drying techniques on the water sorption properties of cement-based materials Cem. Concr. Res. 64 54-62

[42] Zhang J and Scherer G W 2011 Comparison of methods for arresting hydration of cement Cem. Concr. Res. 41 1024-36

[43] Snellings R, De Schepper M, De Buysser K, Van Driessche I and De Belie N 2012 Clinkering reactions 
during firing of recyclable concrete J. Am. Ceram. Soc. 95 1741-9 